\title{
Contents
}

\section{Geometry of State Spaces}

A. Uhlmann, B. Crell ........................ 1

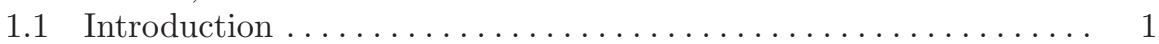

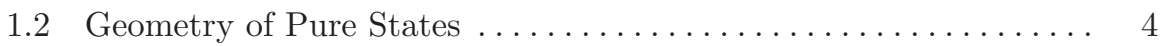

1.2.1 Norm and Distance in Hilbert Space . . . . . . . . . . . . . . 4

1.2.2 Length of Curves in $\mathcal{H} \ldots \ldots \ldots \ldots \ldots \ldots \ldots \ldots \ldots \ldots \ldots \ldots \ldots \ldots$

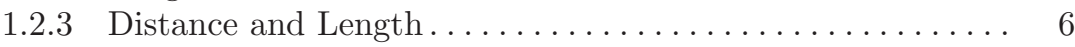

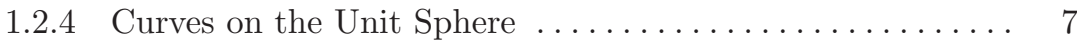

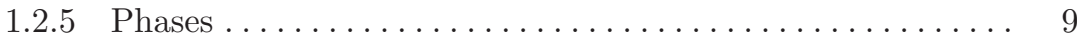

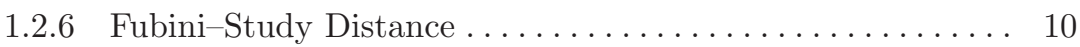

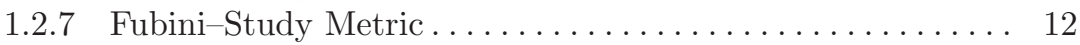

1.2 .8 Symmetries.......................... 13

1.2.9 Comparison with Other Norms................ 15

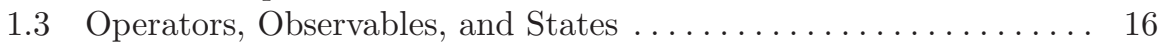

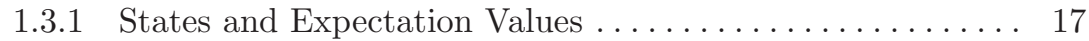

1.3.2 Subalgebras and Subsystems . . . . . . . . . . . . . 19

1.3.3 Classification of Finite Quantum Systems ........... 23

1.3.4 All Subsystems for $\operatorname{dim} \mathcal{H}<\infty \ldots \ldots \ldots \ldots \ldots \ldots \ldots \ldots$

1.4 Transition Probability, Fidelity, and Bures Distance........... 35

1.4 .1 Purification......................... 36

1.4.2 Transition Probability and Fidelity ............ 37

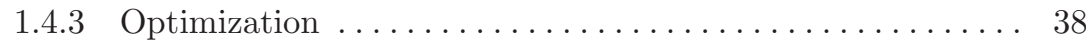

1.4.4 Why the Bures Distance Is a Distance . . . . . . . . . . . 40

1.4.5 Expressions for Fidelity and Transition Probability ..... . 43

1.4.6 Estimates and a "Hidden Symmetry" . . . . . . . . . . . . 47

1.4.7 "Operational Fidelity" . . . . . . . . . . . . . . . . . . . 49

1.5 Appendix: The Geometrical Mean ................... 50

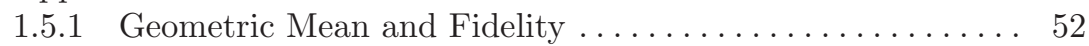

1.5.2 The Transformer Identity . . . . . . . . . . . . . . 53

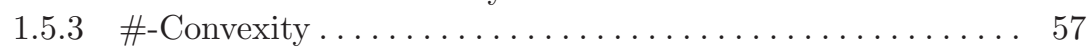

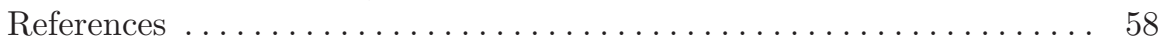




\section{Basic Concepts of Entangled States}

F. Mintert, C. Viviescas, A. Buchleitner ................. 61

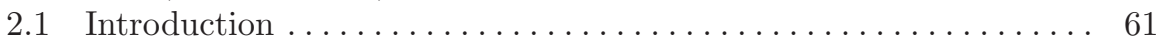

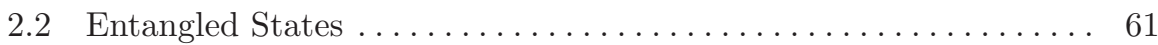

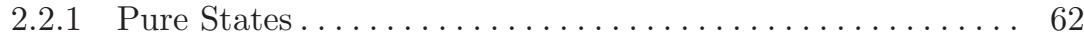

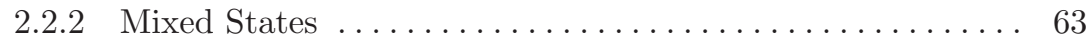

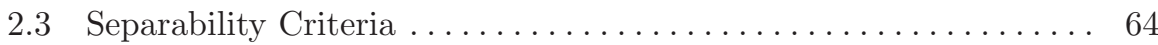

2.3 .1 Pure States.......................... 64

2.3 .2 Mixed States . . . . . . . . . . . . . . . . . . 66

2.4 Entanglement Monotones and Measures . . . . . . . . . . . . . 71

2.4.1 General Considerations .................. 71

2.4.2 Some Specific Monotones and Measures .............. 78

References ............................ 86

\section{Topology and Quantum Computing}

L.H. Kauffman, S.J. Lomonaco Jr. . . . . . . . . . . . . . . . . . . 87

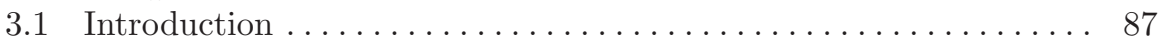

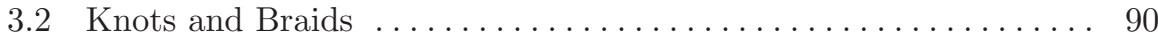

3.3 Quantum Mechanics and Quantum Computation ............. 94

3.3.1 What Is a Quantum Computer?................. 95

3.4 Braiding Operators and Universal Quantum Gates............ 96 3.4 .1 Universal Gates . . . . . . . . . . . . . . . . . . 99

3.5 A Remark About EPR, Entanglement and Bell's Inequality . . . . . 102

3.6 The Aravind Hypothesis . . . . . . . . . . . . . . . . . . . . . . 104

$3.7 S U(2)$ Representations of the Artin Braid Group . . . . . . . . . . . . . . 104

3.8 The Bracket Polynomial and the Jones Polynomial . . . . . . . . . 110

3.8.1 The State Summation ...................... 113

3.8.2 Quantum Computation of the Jones Polynomial . . . . . . . . . 114

3.8.3 The Hadamard Test . . . . . . . . . . . . . . . . . . . . . . 117

3.9 Quantum Topology, Cobordism Categories, Temperley-Lieb

Algebra and Topological Quantum Field Theory ........... 118

3.10 Braiding and Topological Quantum Field Theory . . . . . . . . . . . 127

3.11 Spin Networks and Temperley-Lieb Recoupling Theory . . . . . . 135

3.11 .1 Evaluations . . . . . . . . . . . . . . . . . . . . . . 140

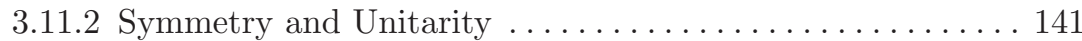

3.12 Fibonacci Particles . . . . . . . . . . . . . . . . . . . . . . . 145

3.13 The Fibonacci Recoupling Model . . . . . . . . . . . . . . . . . . 148

3.14 Quantum Computation of Colored Jones Polynomials and the Witten-Reshetikhin-Turaev Invariant . . . . . . . . . . . . . . . . . . . 150

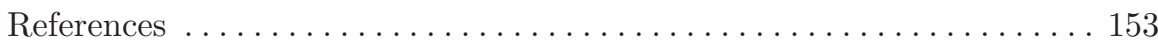

\section{Entanglement in Phase Space}

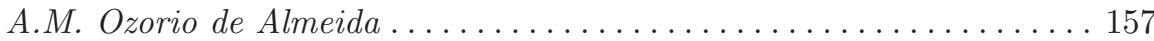

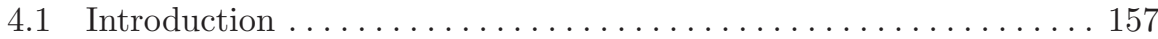

4.2 Entanglement and Classical Physics.................. 160 
4.3 Classical-Quantum Correspondence................... 163

4.4 Semiclassical Quantum States . . . . . . . . . . . . . . . . 169

4.5 Operator Representations and Double Phase Space . . . . . . . . . 175

4.6 The Wigner Function and the Chord Function . . . . . . . . . . . . . 182

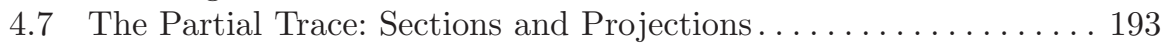

4.8 Generating a Classical Entanglement: The EPR State... . . . . . . 200

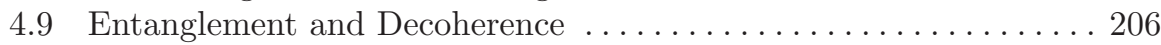

4.10 A Semiclassical Picture of Entanglement . . . . . . . . . . . . 208

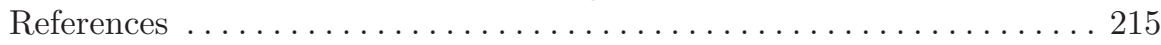

\section{Introduction to Decoherence Theory}

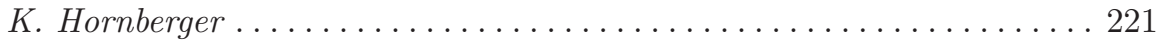

5.1 The Concept of Decoherence .................... 221

5.1 .1 Decoherence in a Nutshell . . . . . . . . . . . . . . . 222

5.1 .2 General Scattering Interaction . . . . . . . . . . . . . . 224

5.1.3 Decoherence as an Environmental Monitoring Process . . . . . 225

5.1 .4 A Few Words on Nomenclature . . . . . . . . . . . . . . 229

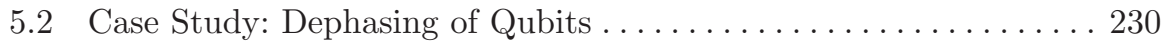

5.2 .1 An Exactly Solvable Model . . . . . . . . . . . . . . . 230

5.2 .2 The Continuum Limit . . . . . . . . . . . . . . . . 234

5.2 .3 Dephasing of $N$ Qubits . . . . . . . . . . . . . . 237

5.3 Markovian Dynamics of Open Quantum Systems . . . . . . . . . . . 239

5.3 .1 Quantum Dynamical Semigroups ................ 240

5.3 .2 The Lindblad Form . . . . . . . . . . . . . . . . . . . . . 242

5.3 .3 Quantum Trajectories...................... 245

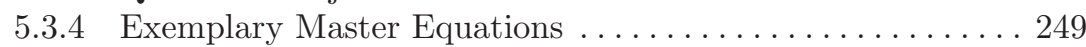

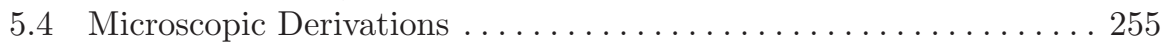

5.4 .1 The Weak Coupling Formulation . . . . . . . . . . . . 255

5.4 .2 The Monitoring Approach . . . . . . . . . . . . . . . 259

5.4 .3 Collisional Decoherence of a Brownian Particle . . . . . . . . 262

5.4 .4 Decoherence of a Quantum Dot ................ 267

5.5 Robust States and the Pointer Basis . . . . . . . . . . . . 270

5.5.1 Nonlinear Equation for Robust States........... . . . 271

5.5 .2 Applications ............................ . 273

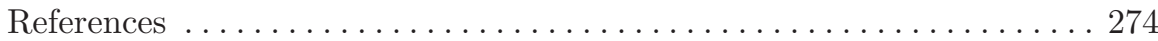

\section{Diffusive Spin Transport}

C.A. Müller................................... 277

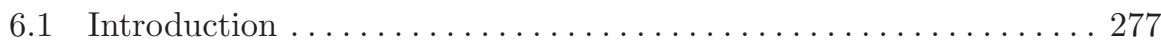

6.2 Spin Relaxation ......................... 278

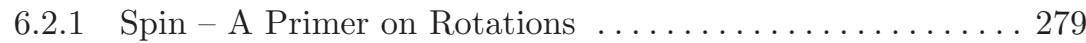

6.2.2 Master Equation Approach to Spin Relaxation . . . . . . . . . . 282

6.2.3 Irreducible Scalar Spin Relaxation Rates . . . . . . . . . . . 287

6.3 Diffusion ................................. 294

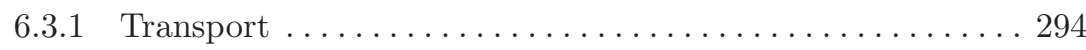


X Contents

6.3.2 Momentum - a Primer on Translations. . . . . . . . . . . . . 295

6.3.3 Master Equation Approach to Diffusion .............. . 296

6.3.4 Linear Response and Diffusion Constant ............. 303

6.4 Diffusive Spin Transport . . . . . . . . . . . . . . . . 306

6.4.1 Master Equation Approach to Diffusive Spin Transport . . 306

6.4 .2 Quantum Corrections ..................... 309

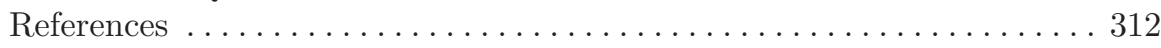

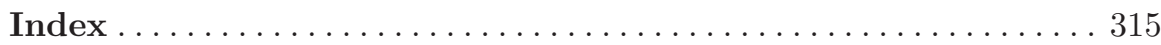

